\title{
Deformation Processing of Sheet Metals by Continuous Frictional Angular Extrusion
}

\author{
Y. Huang ${ }^{1,2, a}$, and P. B. Prangnell ${ }^{2, b}$ \\ ${ }^{1}$ Confae Technology Ltd, 30 Frieston Road, Altrincham, Cheshire WA14 5AP, UK \\ ${ }^{2}$ Manchester Materials Science Centre, the University of Manchester, Grosvenor Street, \\ Manchester M17HS, UK \\ ayan.huang@confae.com, bphilps.prangnell@manchester.ac.uk,
}

Keywords: Deformation processing, continuous frictional angular extrusion (CFAE), sheet metals, deformation structure, texture, ultra-fine grained (UFG) structure, EBSD.

\begin{abstract}
The feasibility of a novel continuous severe plastic deformation (SPD) technique, continuous frictional angular extrusion (CFAE), for producing ultra-fine grained strip material, has been studied. The CFAE technique takes advantage of facets of rolling and equal channel angular extrusion (ECAE) and is designed to produce bulk ultra-fine grained (UFG) metals with high productivity and low cost. A process setup was established through the modification of a standard rolling mill. CFAE processing of commercially pure aluminium AA1050 sheets was successfully carried out at room temperature, using a $120^{\circ}$ die angle. A uniform UFG structure with an average grain size of $\sim 0.6 \mu \mathrm{m}$ was achieved after $10 \mathrm{CFAE}$ passes, at an equivalent strain of $\sim 6.6$. Evolution of the deformation structure and texture during processing was examined as a function of strain and characterized using high resolution EBSD.
\end{abstract}

\section{Introduction}

Severe plastic deformation (SPD) has proved to be an effective method for producing ultra-fine grained (UFG) metals and alloys with enhanced mechanical and physiochemical properties [1,2]. In the last decade or so, extensive research has been carried out in order to develop and optimize SPD techniques for commercial utilization. So far, of all the SPD techniques, equal channel angular extrusion (ECAE) [3] has attracted the most attention, due to its capability of producing UFG structures at scales large enough for structural applications [4,5]. However, the ECAE technique in its original form has disadvantages for commercial application. These include: 1) limited scalability, 2) a high scrap rate, as a significant length near each billet end contains a non-uniform microstructure and has to be removed, and 3) high load requirements. These limitations, plus its batch nature, make ECAE a technique that performs at low production efficiency.

Continuous processing is the key for a commercially viable SPD method that can produce large quantities of UFG metals at high productivity. Several attempts have been made to transform ECAE into a continuous process. For example, continuous constrained strip shearing (C2S2) [6] and ECAP-Conform [7] are recently reported methods for continuously processing sheets and rods to produce UFG structures. A common feature of these processes is the adoption of the Conform technique [8] for feeding the work-piece by friction into a modified ECAE die assembly. Although these techniques exhibit some useful features, they all use a long confined feeding passage for building up pressure on the work-piece to generate sufficient frictional force to push material through the die assembly. This causes several problems. The work-piece undergoes a reduction in thickness, or shape change, before entry into the die's shear zone and consequently, experiences a complicated deformation mode and redundant strains and there is a resultant excessive requirement for torque. Furthermore these techniques have difficulties in lubrication. These problems impose practical limitations for scale-up.

The purpose of the present work was to study experimentally the feasibility of a novel SPD method, called continuous frictional angular extrusion (CFAE), which was developed based on a 
pending UK patent [9], for producing large volumes of UFG structures at high productivity and low cost. The CFAE technique (illustrated in Fig. 1a) involves the use of an ECAE based die assembly combined with several innovative steps, including the use of a frictional driving roll, which applies a normal pressure to the work-piece without any plastic compression, and reduced friction within the die. Thus, continuous processing is possible with plastic deformation occurring principally by shear without changing the dimensions of the work-piece. Ultra-high strains can be achieved through repetition of the process. The CFAE technique offers several benefits over existing methods including: 1) being suitable for processing large quantities of material (including sheet, bar and rod), 2) high productivity and a low scrap rate, and 3) less extrusion force due to minimized die friction and the elimination of the work-piece plastic compression in the first channel. This paper will focus on an investigation into the feasibility of the CFAE technique for processing work-pieces in a sheet form, in terms of operation performance and effectiveness of grain refinement, using a modified rolling mill.
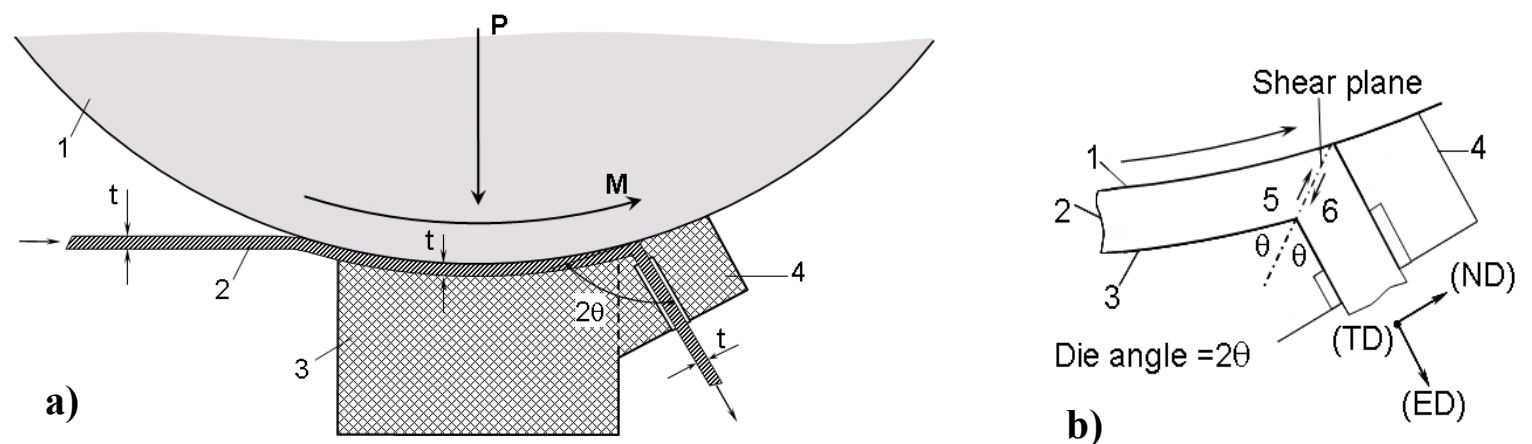

Fig. 1. a) Schematic diagram of the CFAE setup: 1-driving roll; 2-sheet work-piece, 3- work-piece support block, 4-die assembly, where $2 \theta$ is the extrusion angle; b) Schematic diagram showing the relationship between extrusion channels and shear plane. The reference system used to describe textures is also shown: ED- extrusion direction, ND-normal to the sheet, and TD-transverse direction.

\section{Experimental procedures}

Process setup. A two-high rolling mill was adapted for CFAE processing of sheet samples, as illustrated in Fig. 1a. The top roll remained untouched and functioned as the driving roll (1) rotating to provide a torque $(\mathrm{M})$ and at the same time applying a pressing force $(\mathrm{P})$ to the work-piece (2). A work-piece support block (3) with an attached die assembly (4) was used as replacing the bottom roll. The working surface of the work-piece support block was matched with the curvature of the sheet on the driving roll and formed the first extrusion channel (5). The second extrusion channel (6) was a short slot, to minimize friction, in the stationary die assembly, and is enlarged in diagram Fig. 1a, illustrating the relationship between the extrusion channels and shear plane. The rotating roll also reduces the friction in the first die channel. The second extrusion channel was inclined at an extrusion angle of $2 \theta$. Both extrusion channels had an identical cross section which matched the sheet thickness. Similar to ECAE, under ideal simple shear conditions the effective plastic strain $\left(\varepsilon_{\mathrm{vm}}\right)$ that the work-piece undergoes through the die assembly is dependent only on the extrusion angle $(2 \theta)$ and the extrusion pressure $(p)$ is a function of the material strength $\left(\sigma_{\mathrm{s}}\right)$ and the extrusion angle, as given below.

$$
\varepsilon_{v m}=1.15 \cot \theta
$$

$$
p=1.15 \sigma_{s} \cot \theta
$$

Material and processing conditions. $2 \mathrm{~m} \times 30 \mathrm{~m}$ samples of $2 \mathrm{~mm}$ thick cold rolled commercial purity aluminum AA1050 sheet were used for CFAE processing, cut in the rolling direction. Processing was carried out at room temperature at 0.09 and $0.2 \mathrm{~m} / \mathrm{s}$. The sheets were processed to up to ten passes (equivalent true strain of 6.6) with the sheet orientation maintained constant throughout. In order to ensure that the frictional coefficient between the driving roll and the work- 
piece was significantly larger than that between the work-piece and the support block, which is an essential requirement for the functioning of the process, a diamond-like carbon coating was applied to the surfaces of the support block and the second extrusion channel and the driving roll was chemically and mechanically roughened. A combination of PTFE spray and colloidal graphite were used for lubricating the bottom surface of the work-piece. The top surface of the work-piece, which was in contact with the driving roll, underwent cleaning to ensure sufficient frictional force for extrusion.

Examination of deformation structures and textures. The deformation structures of the CFAE processed sheets were examined in a FEG-SEM and using high resolution EBSD on the longitudinal ND-ED/RD plane (see Fig. 1b) after mechanical and electropolishing, to give a strain-free surface. EBSD orientation maps were acquired at different strain levels with a spatial and angular resolution of $\sim 50 \mathrm{~nm}$ and $\sim 1^{\circ}$. EBSD data was analysed by VMAP $\odot$ software. In the data presented, highangle boundaries (HABs) and low-angle boundaries (LABs) are defined as having misorientations greater than, or equal to, $15^{\circ}$ or less than $15^{\circ}$. Due to misorientation noise boundaries of less than $1.5^{\circ}$ misorientation were cut off. Crystallographic textures were determined by EBSD from a minimum of 10,000 orientations over an area of $500 \times 500 \mu \mathrm{m}$.

\section{Experimental results and discussions}

General performance. Using the setup illustrated in Fig. 1, CFAE processing performed successfully under a range of conditions. The use of a diamond-like carbon coating and a Colloidal graphite/PTFE lubricant on the stationary tool surfaces was found to reduce the friction coefficient sufficiently to obtain the friction differential required for extrusion. Deformation was found to be reasonably uniform over the cross section of the work-piece and the effects of surface friction were limited (Fig. 2). The friction affected zone was deeper $(\sim 100 \mu \mathrm{m})$ at the stationary die bottom surface than the top roll contacted surface (Fig. 2a, c), where the microstructure was almost indistinguishable from the matrix (Fig. 2b, d). This phenomenon is a characteristic feature of CFAE, due to fact that the bottom surface experiences sliding contact with the support block, while the top surface moves at the same speed as the driving roll. With more passes the frictional control required for processing was found to be less demanding, although the extrusion force initially increased due to a rise in material strength, it thus seems that the frictional coefficient decreased with increase in material hardness.

Deformation structure evolution and grain refinement. Example EBSD maps are shown in Fig.3, illustrating the evolution of the deformation structure with strain. High resolution EBSD statistical data (Fig. 4) revealed that a significant fraction of high angle boundaries (HABs) developed in the deformation structure after 4 CFAE passes and HABs became dominant after 8 CFAE passes, with a fraction of $65 \%$, and a final fraction of $\sim 70 \%$ was obtained at a strain of $\sim 6.6$. The starting material was in a commercially cold rolled state, having a mean "grain size" of $\sim 35 \mu \mathrm{m}$ in the matrix of a coarse subgrain structure. After 1 CFAE pass, a uniform and finer subgrain structure formed from the initial coarse cold-rolled structure with cell bands aligned along the shear plane, which is $60^{\circ}$ to the vertical extrusion direction. Evidence of deformation banding, which is usually expected to occur in the early stages of ECAE processing, was not observed, most likely due to the use of a cold rolled starting material. With further passes a fibrous deformation structure started to form along a direction between the die shear plane and the extrusion direction, although aligned cell bands were still visible up to 2 passes. 
a)

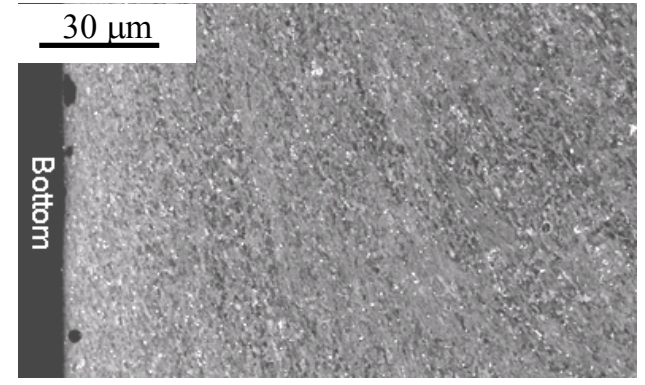

c)

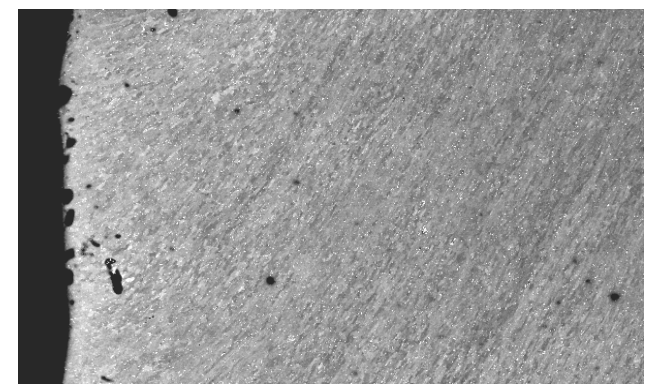

b)

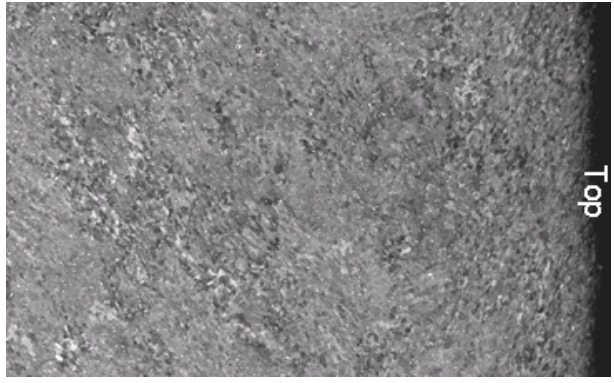

d)

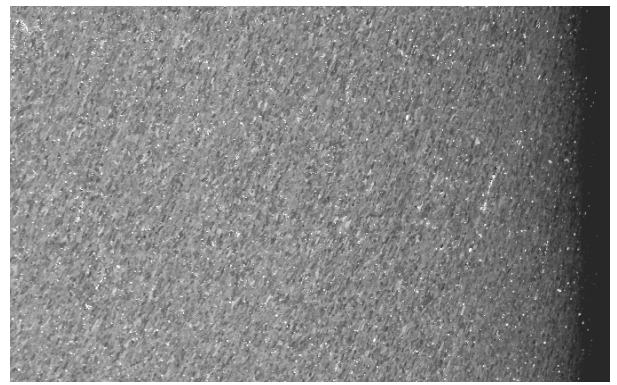

Fig. 2 FEGSEM backscattered images showing the effect of surface friction on deformation structures: a) bottom surface, 1 pass; b) top surface, 1 pass; c) bottom surface, 8 passes; d) top surface, 8 passes. The extrusion direction (ED) is down vertically.

a)

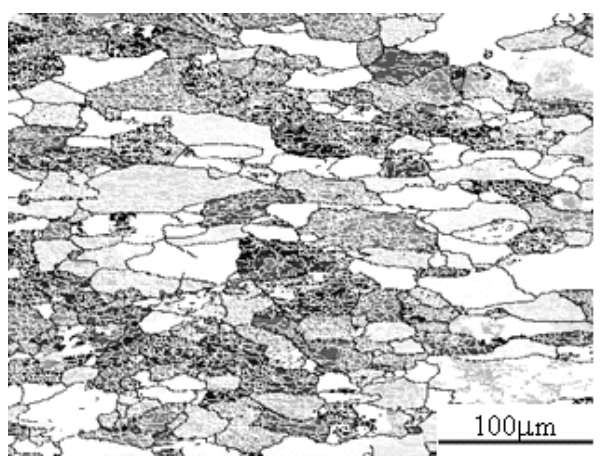

c)

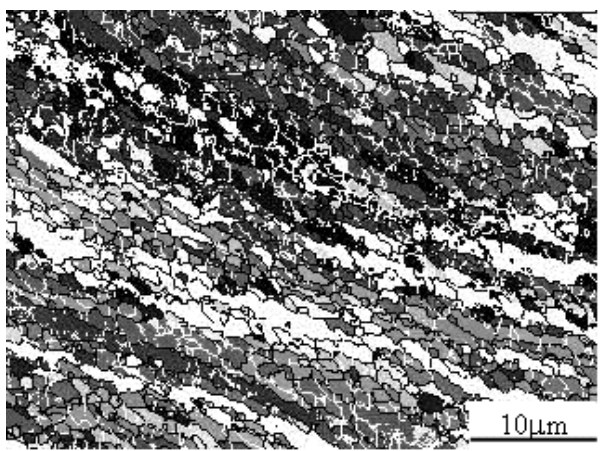

b)

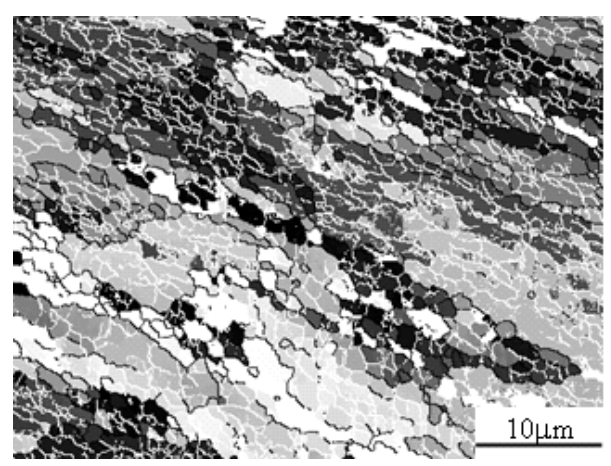

d)

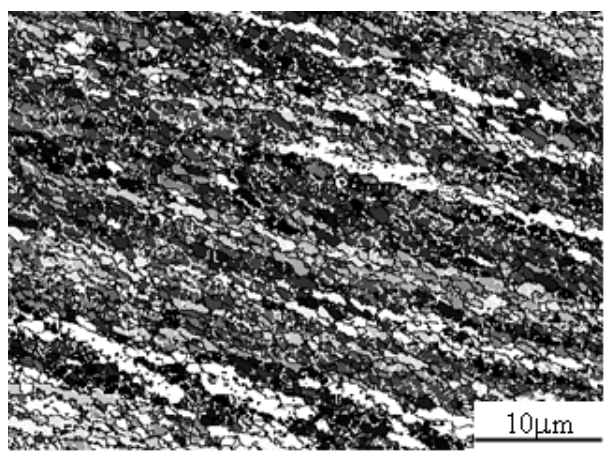

Fig. 3. EBSD maps of CFAE processed AA1050 sheet samples, showing evolution of deformation structure with strain: a) 0 (commercially cold rolled); b) $\varepsilon_{\mathrm{vm}} \sim 2.7$. (4 passes); c) $\varepsilon_{\mathrm{vm}} \sim 5.3$ (8passes) and d) $\varepsilon_{\mathrm{vm}} \sim 6.6$ (10 passes). Black lines represent high angle grain boundaries $\left(>15^{\circ}\right)$ and white lines low angle grain boundaries $\left(2-15^{\circ}\right)$. Extrusion direction (ED) is approximately horizontally aligned in all the maps.

With increasing strain, a lamellar HAB fibrous structure developed, which compressed in transverse spacing, became more uniform, and rotated towards the extrusion direction (Fig. 3b-c). After 8 CFAE passes $\left(\varepsilon_{\mathrm{vm}} \sim 5.3\right)$ the average spacing of HABs, or grain width, was at a submicron scale and in the most regions groups of pancake-like submicorn grains were seen to have formed within the lamellar structure. After 10 CFAE passes $\left(\varepsilon_{\mathrm{vm}} \sim 6.6\right.$; Fig. $\left.4 \mathrm{~d}\right)$ the deformation structure can be seen to have developed into a reasonably uniform submicron, or UFG structure, with an average grain size of $0.6 \mu \mathrm{m}$, although it still contained some unrefined bands. The final fraction of 
HAB area reached was $70 \%$, which is sufficient to form a stable HAGB network without discontinuous recrystallization on annealing [10]. This occurred at slightly lower strain than that expected from conventional ECAE processing of a comparable high purity model alloy [11], however, the supplied material was already in an $\mathrm{H}$ temper. Nevertheless, the overall trends of statistical data are in agreement with previous ECAE studies [11] and suggest that while the grain width has achieved a steady state value after 10 passes, the fraction of HAB area still has some potential to increase, and grain aspect ratio to reduce, upon further deformation, with typical levels of $\mathrm{HAB}$ fraction saturating at $\sim 80 \%$ in ECAE processing.

Fig. 4. Statistical data from EBSD maps of CFAE processed sheet samples, showing grain width, aspect ratio and the percentage of HAGB area as a function of CFAE pass of up to $10\left(\varepsilon_{\mathrm{vm}} \sim 6.6\right)$. Note grain size data for the starting material have been omitted for clarity.

a)

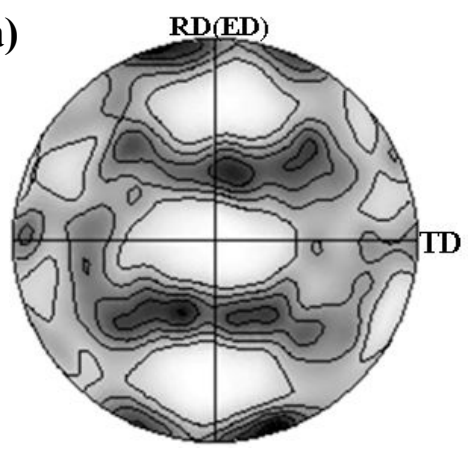

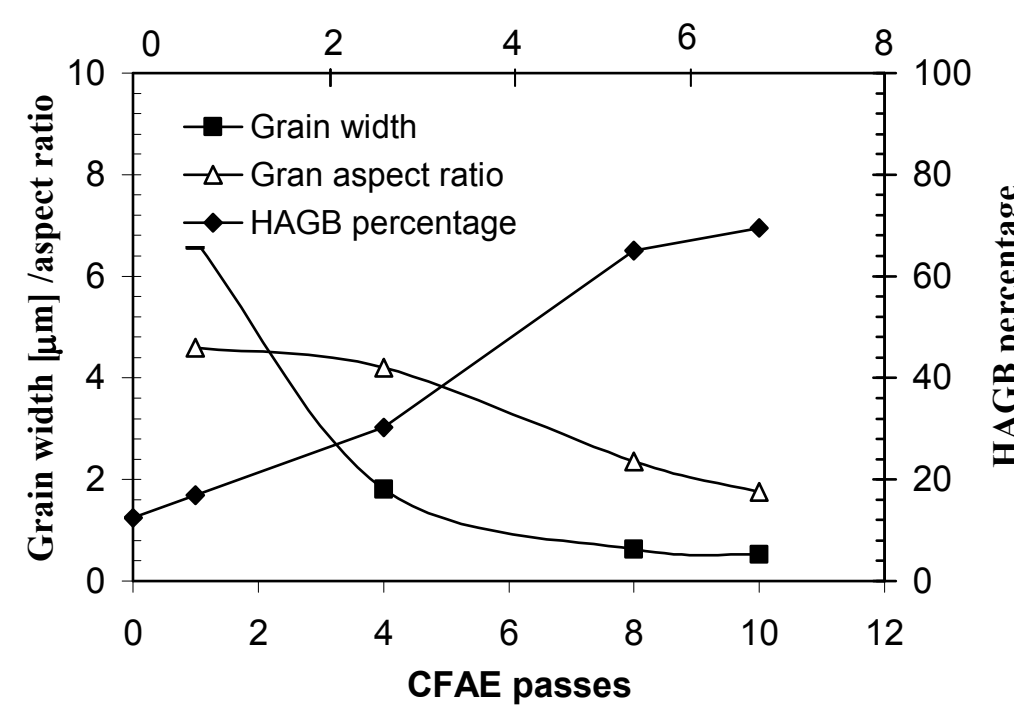
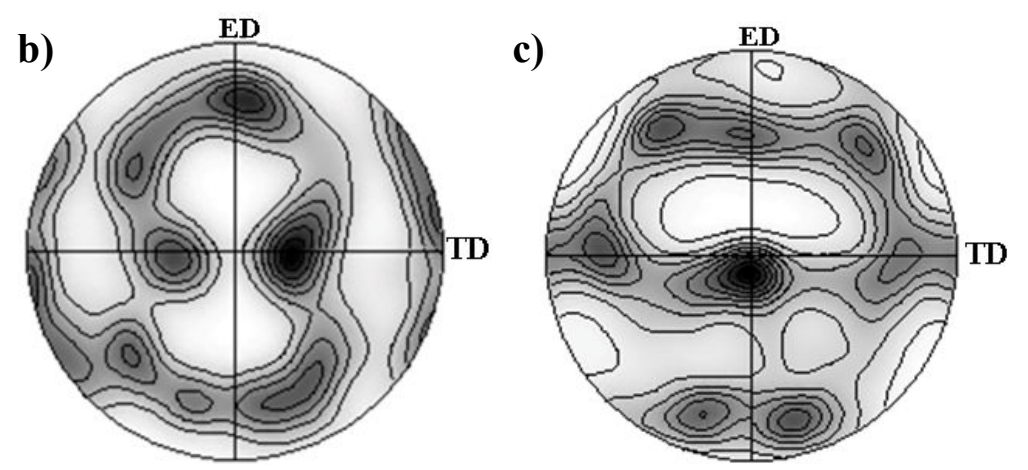

Fig. 5. 111 pole figures showing the texture evolution with CFAE strains; a) the initial rolling texture and b) after 1 pass, and c) 8 passes.

Texture Development. Following deformation, crystallographic textures of samples processed to different numbers of CFAE passes were determined by EBSD. Fig. 5 shows 111 pole figures of the starting material and the deformation textures after 1 , and 8 CFAE passes. The initial rolling texture was comprised primarily of a mixture of strong $S\{123\}<634>$ and Brass $\{011\}<112>$, and relatively weaker Cube $\{001\}<100>$ and $\mathrm{P}\{011\}<122>$ components (Fig. 5a). After 1 CFAE pass, the initial texture components weakened as shown in Fig. 5b, and with reference to the s sheet plane $\}<$ ED $>$ a simple shear texture $\{111\}<110>$ and rotated cube component $\{100\}<110>$ began to form. This evolution continued with increasing strains and after 4 CFAE passes the initial texture disappeared. After $8 \mathrm{CFAE}$ passes, textures associated with CFAE deformation developed. The main texture components were, approximately, shear texture $\{111\}<110>$, rotated cube $\{100\}<110>$, S $\{123\}<634>$ and weak cube $\{001\}<100>$, which are broadly similar to findings in fcc metals processed by ECAE [12] and by C2S2 [13]. 
It is well known that the formability of metal sheets is texture dependent. Textures with $\{111\}$ planes parallel to the sheet plane enhances formability; whereas textures with $\{001\}$ planes parallel to the sheet plane does the opposite. In this sense, the development of a $\{111\}<110>$ shear texture and the weakening of the cube texture $\{001\}<100>$ during the present CFAE processing are advantageous because they will help to improve the formability of the material.

\section{Summary}

This experimental feasibility study has shown that continuous frictional angular extrusion is an effective method for producing UFG metal sheets at high productivity and low cost, although more investigation is needed to verify its advantageous features with larger scale processing. The study demonstrated that a two-high rolling mill can be used for CFAE processing, after only moderate modification. CFAE processing of AA1050 sheet samples was successfully carried out to various total numbers of passes and a uniform and nearly equaixed UFG structure was achieved after 10 CFAE cycles, or at an equivalent strain of 6.6. The evolution of deformation structure and texture during CFAE processing was examined using the EBSD technique and was found to be similar to that seen in ECAE. The developed textures exhibited advantageous features in terms of improving formability of metal sheets.

\section{Acknowledgements}

The authors would like to acknowledge the financial support of the Department of Trade and Industry, UK (GONW8080TEC), and the University of Manchester EPSRC Light Alloys Portfolio Partnership (EP/D029201/1) for this project.

\section{References}

[1]. R.Z. Valiev, N.A. Krasilnikov and N.K. Tsenev: Mater. Sci. Eng. Vol. A137 (1991), p. 35.

[2]. F.J. Humphreys, P.B. Prangnell, J.R. Bowen, A. Gholinia and C. Harris: Phil Trans R Soc Lond. Vol. A 357 (1999), p. 1663.

[3]. V.M. Segal: Mater. Sci. Eng. Vol. A197 (1995), p. 157.

[4]. R.Z. Valiev, R.K. Islamgaliev and I.V. Alexandrov: Prog. Mater. Sci. Vol. 45 (2000), p. 103

[5]. V. M. Segal: Mater. Sci. Eng. Vol. A386 (2004), p. 269.

[9]. Y. Saito, H. Utsunomiya, H. Suzuki and T. Sakai: Scripta Mater. Vol. 42 (2000), p. 1139.

[6]. J.C. Lee, H.K. Seok and J.Y. Suh: Acta Mater. Vol. 50 (2002), p. 4005

[7]. J. Georgy et al: Mater. Sci. Eng. Vol. A382 (2004), p. 30.

[8]. D. Green, Atomic Energy Authority UK: UK patent GB1370894 (1973).

[9]. Y. Huang: UK patent GB2490997A (2004).

[10]. P. B. Prangnell et al: Acta Mater Vol. 52 (2004), p. 3193.

[11]. P. B. Prangnell, J. R. Bowen and P. Apps: Mater. Sci. Eng. Vol. A373-377 (2004), p. 178.

[12]. W. Huang, L. Chang, P. W. Hao and C. P. Chang: Mater. Sci. Eng. Vol. A307 (2001), p. 87.

[13]. J. -C. Lee et al: Mater. Res. Bullet Vol. 36 (2001), p. 887. 